\title{
ON 3-COLORINGS OF DIRECT PRODUCTS OF GRAPHS
}

\author{
SIMON ŠPACAPAN ${ }^{1}$ \\ University of Maribor, FME, Smetanova 17, 2000 Maribor, Slovenia \\ IMFM, Jadranska 19, 1000 Ljubljana, Slovenia \\ e-mail: simon.spacapan@um.si
}

\begin{abstract}
The $k$-independence number of a graph $G$, denoted as $\alpha_{k}(G)$, is the order of a largest induced $k$-colorable subgraph of $G$. In [S. Špacapan, The $k$-independence number of direct products of graphs, European J. Combin. 32 (2011) 1377-1383] the author conjectured that the direct product $G \times H$ of graphs $G$ and $H$ obeys the following bound

$$
\alpha_{k}(G \times H) \leq \alpha_{k}(G)|V(H)|+\alpha_{k}(H)|V(G)|-\alpha_{k}(G) \alpha_{k}(H),
$$

and proved the conjecture for $k=1$ and $k=2$. If true for $k=3$ the conjecture strenghtens the result of El-Zahar and Sauer who proved that any direct product of 4-chromatic graphs is 4-chromatic [M. El-Zahar and N. Sauer, The chromatic number of the product of two 4-chromatic graphs is 4, Combinatorica 5 (1985) 121-126]. In this paper we prove that the above bound is true for $k=3$ provided that $G$ and $H$ are graphs that have complete tripartite subgraphs of orders $\alpha_{3}(G)$ and $\alpha_{3}(H)$, respectively.
\end{abstract}

Keywords: independence number, direct product, Hedetniemi's conjecture.

2010 Mathematics Subject Classification: 05C15, 05C69, 05 C67.

\section{REFERENCES}

[1] N. Alon, I. Dinur, E. Friedgut and B. Sudakov, Graph products, Fourier analysis and spectral techniques, Geom. Funct. Anal. 14 (2004) 913-940. doi:10.1007/s00039-004-0478-3

[2] M. El-Zahar and N. Sauer, The chromatic number of the product of two 4-chromatic graphs is 4, Combinatorica 5 (1985) 121-126. doi:10.1007/BF02579374

\footnotetext{
${ }^{1}$ The author is supported by ARRS, grant P1-0297.
} 
[3] D. Greenwell and L. Lovász, Applications of product colouring, Acta Math. Acad. Sci. Hungar. 25 (1974) 335-340.

doi:10.1007/BF01886093

[4] X.B. Geng, J. Wang and H. Zhang, Structure of independent sets in direct products of some vertex-transitive graphs, Acta Math. Sin. (Engl. Ser.) 28 (2012) 697-706. doi:10.1007/s10114-011-0311-5

[5] R. Hammack, W. Imrich and S. Klavžar, Handbook of Product Graphs, Second Edition (CRC Press, Boca Raton, FL, 2011).

[6] S.T. Hedetniemi, Homomorphisms and graph automata, University of Michigan Technical Report 03105-44-T (1966).

[7] P.K. Jha and S. Klavžar, Independence in direct-product graphs, Ars Combin. 50 (1998) 53-63.

[8] B. Larose and C. Tardif, Projectivity and independent sets in powers of graphs, J. Graph Theory 40 (2002) 162-171.

doi:10.1002/jgt.10041

[9] S. Špacapan, The k-independence number of direct products of graphs and Hedetniemi's conjecture, European J. Combin 32 (2011) 1377-1383. doi:10.1016/j.ejc.2011.07.002

[10] C. Tardif, Hedetniemi's conjecture 40 years later, Graph Theory Notes of New York LIV (2008) 46-57.

[11] C. Tardif, Hedetniemi's conjecture and dense Boolean lattices, Order 28 (2011) $181-191$. doi:10.1007/s11083-010-9162-4

[12] Á. Tóth, On the ultimate categorical independence ratio, J. Combin. Theory Ser. B 108 (2014) 29-39. doi:10.1016/j.jctb.2014.02.010

[13] M. Valencia-Pabon and J. Vera, Independence and coloring properties of direct products of some vertex-transitive graphs, Discrete Math. 306 (2006) 2275-2281. doi:10.1016/j.disc.2006.04.013

[14] H. Zhang, Primitivity and independent sets in direct products of vertex-transitive graphs, J. Graph Theory 67 (2011) 218-225. doi:10.1002/jgt.20526

[15] H. Zhang, Independent sets in direct products of vertex-transitive graphs, J. Combin. Theory Ser. B 102 (2012) 832-838. doi:10.1016/j.jctb.2011.12.005

[16] X. Zhu, A survey on Hedetniemi's conjecture, Taiwanese J. Math. 2 (1998) 1-24. doi: $10.11650 /$ twjm/1500406890

[17] X. Zhu, The fractional version of Hedetniemi's conjecture is true, European J. Combin. 32 (2011) 1168-1175. doi:10.1016/j.ejc.2011.03.004 
Received 17 November 2016

Revised 25 May 2017

Accepted 11 September 2017 